\title{
THE HIDDEN FEAT BEHIND DEVELOPMENT COST ESCALATION - HOW ENGINEERING DESIGN ENABLES FUNCTIONAL EXPANSION IN THE AEROSPACE INDUSTRY
}

\author{
Gilain, Agathe; Le Masson, Pascal; Weil, Benoît \\ MINES ParisTech
}

\begin{abstract}
The aerospace industry experiences a considerable growth in product development costs. Many research works aim at identifying evolution laws characterizing this large-scale phenomenon and at developing design strategies which could help mitigate it. This paper aims to clarify the evolution dynamics governing this phenomenon by studying how the products delivered by these costly projects evolve with time. Increasing complexity is often held responsible for surging costs. If complexity is generally defined as the price to be paid for improving product functionalities, it is rarely specified whether the improvement affects existing functionalities or involves new ones. We aim to identify the patterns of cost growth which can be associated with phenomena of existing functionalities upgrade and new functionalities introduction, and to identify the associated design capabilities that designers need to deploy in order to keep product change and cost growth under control. To that end, we introduce a model which generates curves, each of which featuring a trend of cost growth, specific to a scenario of product evolution and being interpretable as a signature of a strategy used by designers.
\end{abstract}

Keywords: Design costing, Functional modelling, Product architecture, Innovation, Functional expansion

\section{Contact:}

Gilain, Agathe

MINES ParisTech

CGS

France

agathe.gilain@mines-paristech.fr

Cite this article: Gilain, A., Le Masson, P., Weil, B. (2019) 'The Hidden Feat Behind Development Cost Escalation - How Engineering Design Enables Functional Expansion in the Aerospace Industry', in Proceedings of the 22nd International Conference on Engineering Design (ICED19), Delft, The Netherlands, 5-8 August 2019. DOI:10.1017/ dsi.2019.308 


\section{INTRODUCTION}

Over the last decades, the aerospace industry has been experiencing a considerable increase in product development costs. A famous example of this phenomenon is Norman Augustine's (1983) law (The Final Law of Economic Disarmament), according to which, the entire American defence budget will be consumed from buying one military aircraft by 2054, if the increasing trend persists. Commercial aircraft development programs are also subject to a phenomenon of cost escalation, as steadily increasing development times (from program launch to Entry Into Service (EIS)) reveal (be it in the case of full developments as illustrated in Figure 1 (JMDLV (C) or derivative aircraft). (The development costs associated with increasing development times escalate accordingly).

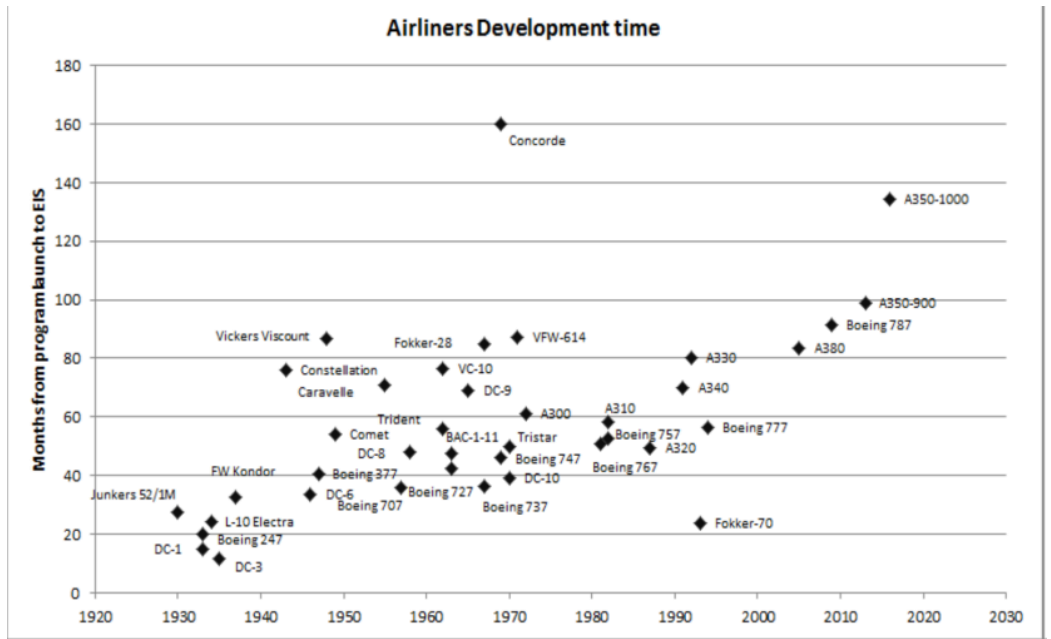

Figure 1. steady growth in commercial aircraft development times (JMDLV ())

Representing a possible threat to the profitability of the projects, programs and even firms associated with these costs (Winter, 2015), this phenomenon appears alarming and symptomatic of engineering departments having lost control over new product development. Therefore, many research works and studies aim at grasping the reasons for this growth, i.e. at identifying the laws, the patterns of the increase, the driving forces... characterizing this large-scale phenomenon (Arena et al., 2008 ; Stuart et al., 2011 ; Hove and Lillekvelland, 2015) and at developing design strategies and tools which could help slow it down and mitigate it (de Weck, 2012).

This paper aims at investigating and characterizing the challenges faced by engineering departments in order to assess the extent of the 'loss of control' at issue. To that end, our approach consists in clarifying the evolution dynamics governing this cost escalation phenomenon by studying how the products, i.e. the outputs delivered by these costly programs and projects evolved with time.

Section 2 reviews the laws characterizing product evolution dynamics, in terms of complexity (Carlson and Doyle, 1999, 2005 ; Sinha, 2014) and functional expansion (that is an increase in the number of functionalities fulfilled by consumption goods) (El Qaoumi, 2016). Our objective is to identify the patterns of cost growth which can be associated with evolution phenomena occurring at product level, as well as the capabilities that designers need to deploy in order to keep these phenomena under control. Therefore, Sections 3 and 4 propose a model which generates the cost growth patterns associated with specific scenarii of product changes implemented with specific design capabilities. Then, in the face of cost growth patterns observed in real cases, this model can diagnose which aspects of product change were more or less kept under control by designers. This enables to (re)interpret cost growth phenomena such as the one presented in introduction. We discuss these results in Section 5.

\section{LITERATURE REVIEW}

This section aims at reviewing the literature related to product evolution dynamics, in an attempt to see how it can help interpret costs escalation phenomena. 


\subsection{Product change and complexity}

Technical systems growing complexity (Spinney, 1980; Carlson and Doyle, 2002; DARPA, 2008; Stuart et al., 2011 ; Sinha, 2014 ; Hove and Lillekvelland, 2015) is widely associated with escalating costs. The notion of increasing complexity plays an important role in product evolution. Indeed, when implementing products changes (specified in the form of modified or new product requirements), designers must handle three major aspects of complexity (uncertainty, interconnectedness, and emergence). Firstly, dealing with complexity involves dealing with uncertainty, since complexity can be defined as "a measure of uncertainty in understanding what we want to know or in achieving a functional requirements (Suh, 2005). According to Suh's information axiom, efficiently handling complexity involves seeking to maximize the probability of satisfying the functional requirements (i.e. minimizing the information content) (information axiom). Dealing with complexity also involves managing interconnectedness, i.e. the interactions between the heterogeneous elements of a system. And according to Suh's (2005) independence axiom, the best design is the one which minimizes the level of coupling between the functional requirements. The notion of complexity also includes a phenomenon called emergence, which involves both uncertainty and interactions. Emergence phenomena (Alderson and Doyle, 2010 ; Carlson and Doyle, 2002) denote to unexpected (and often undesirable) behaviours resulting from unpredicted interactions between some subsystems.

Sinha (2014), Carlson and Doyle (2002) suggest that evolving a product in order to improve its functionalities generally goes together with an increase in complexity, which represents potentially harmful and costly side effects. However, this does not mean that complexity must systematically be avoided. This suggests that designers face a trade-off between 'increased functionality' versus 'harmful side effects and associated costs': indeed, the value to be gained from a functional increased can be worth paying the cost of complexity. (Sinha, 2014 ; Carlson and Doyle, 2002).

Since increasing complexity appears central in product evolution, the next subsection is dedicated to the laws which appear to govern the evolution of complexity, as the product evolves.

\subsection{Product complexity evolution laws}

\subsubsection{A convergence process toward a simplified configuration}

According to some scholars, the evolution dynamic of a product can be seen as a phase of 'complexification' (in which functionalities are improved at the expense of simplicity) followed by a phase of 'simplification' (Salamatov, 1999), that is of convergence toward a simplified and ideal state (Altshuller, 1984). A parallel can be made with technology S-curves (Christensen, 1994) describing the evolution of a product toward a Dominant Design, the first flat phase, at which the product emerges corresponding to an emergence of functionalities and the second flat phase, after the inflexion point, corresponding to a stabilization, with only minor improvements (e.g. processes improvements...) and little change in the product itself. However, other research works suggest that the dynamics of product complexity and product evolution are not as simple as a 'complexification' phase followed by a 'simplification' phase.

\subsubsection{A continuous increase in complexity}

Carlson and Doyle (1999) describe a product dynamics characterized by a continuous complexification of technical systems. Their theory called 'Highly Optimized Tolerance' explains this trend. 'Highly optimized' denotes to systems that are highly performing. 'Tolerances' outline a high level of structuration / organization. HOT is defined in opposition with another complexity evolution dynamic theorized by NSCN (New Sciences of Complexity Networks). NSCN describe complex systems spontaneously evolving following self-organizing rules, which only required one parameter (the density of the systems elements) to be set. Carlson and Doyle (1999) stress that self-organization is suited for problems of disorganized complexity, involving an infinite number of homogenous elements, such as fractals for instance. But it is misleading to use these mechanisms to interpret or model the evolution of technical systems which fall into the problem category of organized complexity, involving a large, but not infinite number of heterogeneous interconnected elements. The elements of these very systems do not self-organize. Quite the opposite, a 'designing force' tunes several design parameters and rigorously arranges the elements of the system into a highly structured and hierarchized organization. 
The evolution dynamics described by Carlson and Doyle is such that systems tend to become more and more robust. Indeed, technical systems are required to operate under increasingly large operating windows (e.g. an aircraft operating under extreme weathers). But as systems get more robust, they also become more sensitive to emergence phenomena (e.g. bugs...). They are designed to handle a larger spectrum of conditions. But the remaining and unpredictable conditions they are not designed for represent increasingly devastating perspectives if they occur. In order to counter emergence phenomena, the 'designing force' builds barriers aiming at preventing any "discussions" between the elements whose interaction represents risky emergence behaviours. Most of these barriers are not obviously detectable, they represent a form of hidden complexity. They keep expanding as the system gets more complex and robust. The concepts of product platforms, product lines, modularity, reuse, commonality (Kalligeros et al., 2006 ; Suh et al., 2004 ; Baldwin and Clark, 2006) propose design strategies aiming at helping designers implementing such barriers in order to keep complexity and costs under control.

These barriers can be interpreted as elements fulfilling new control functionalities in the system. However, HOT theory does not treat the scenario where new functionalities whose intended purpose would not be to prevent fragility but to add an additional characteristic to the system would emerge. The following subsection (2.3) reviews recent research works highlighting that the emergence of such new functionalities can be far from negligible within a technical system.

\subsection{Product functional evolution law}

Recent research works on product functional evolution reveal a steady increase in the emergence of new functionalities which affects consumption goods, such as the toothbrush, the hoover (El Qaoumi, 2016). These works identify a pattern of growth in the number of new functionalities that corresponds to a permanent transformation, involving not rare nor random, but frequent functional disruptions: such patterns of growth are characterized as regimes of functional expansion (El Qaoumi, 2016). If one considers each function as one edge of a graphic matroid, different possible evolution scenarii of the matroid rank can be associated with different regimes of functional expansion (El Qaoumi, 2016 ; Le Masson et al., 2018). One of these regimes is called 'endogenous expansion' where endogenous means that the functional change does not result from exogenous / external events (e.g. market-pull or techno-push dynamics, regulatory requirements...) but from a dynamic of design that is internal to the product. Empirical tests reveal that most of the studied consumption goods (apart from the refrigerator) follow this regime of endogenous expansion (El Qaoumi, 2016) which accelerates from the 1990s (Le Masson et al., 2018). These findings contrast with Lancaster's (1966) theory according to which the evolution of goods results from the combinations and re-combinations of a given fixed set of characteristics. Here, functional transformation takes place within a space which is in permanent expansion.

\subsection{Research questions}

Subsections 2.2 and 2.3 highlight the existence of two large-scale phenomena ((i) an explosion in robustness and (ii) an explosion in the number of functionalities) occurring at product level. These seem promising to help characterize the laws governing development cost escalation.

To our best knowledge, there exist no study documenting and representing a possible expansion of product functional space in the aerospace industry, although a few reports and papers (GAO, 2015 ; Dabkowski and Valerdi, 2016) attribute cost increase to the introduction of 'new system capabilities' (i.e. new functionalities), in retrospective studies of projects affected with cost overruns). One can note that most research works associating cost escalation phenomena with an increase in complexity resulting from functional improvements do not specify whether these very functional improvements regard existing functionalities or newly-introduced ones.

This leads to our first research question: Are the products developed in the aerospace industry subject to a phenomenon of functional expansion, governed by an endogenous evolution law? (RQ1).

If the answer to RQ1 is affirmative, our second research question is: How can we model, and hence predict the cost growth pattern stemming from a product change scenario whose implementation is carried out with specific design capabilities? (RQ2)

Finally, our third research question is: To what extent can the cost escalation phenomena occurring within the aviation industry be (re)interpreted by identifying the design strategy revealed by these trends?(RQ3) 


\section{METHOD}

This paper results from investigations carried out within one global commercial aircraft manufacturing company. Therefore, we address RQ1 by testing whether the functional evolution of commercial aircraft is subject to a phenomenon of functional expansion. And in our answer to RQ3, the cost growth patterns which will be tested also come from projects led in the development area of commercial aircraft.

\subsection{Testing the presence of a phenomenon of functional expansion at aircraft level}

For an Aircraft to be certified, it must be demonstrated that its design complies with the Airworthiness Requirements applying at different levels. At aircraft level, the applicable regulation is CS-25 (released by the European regulation Agency, EASA) and FAR-25 (released by the American agency, FAA).

The amendments affecting FAR-25 and CS-25 are particularly interesting for our research. Indeed, two main mechanisms trigger regulatory changes. On the one hand, a change in design can be triggered by a change in the airworthiness requirements, following the report of an unsafe situation (most of the time revealed by incidents / accidents). Conversely, new functionalities or new designs initiated by aircraft manufacturers trigger the enactment of new airworthiness requirements in CS-25 and FAR-25, in order to make the new design certifiable. Therefore, changes in certification requirements record the introduction of new or updated (e.g. reprioritized) intended purposes at aircraft level (i.e. new functionalities). For this reason, with one paragraph of CS-25 / FAR-25 used as a unit, we counted and summed, at the time of each Amendment: (i) the number of added paragraphs, (ii) modified paragraphs, (iii) deleted paragraphs (counted positively), in order to assess the magnitude of the changes affecting commercial aircraft functionalities.

\subsection{Cost growth and design strategies associated with robustness explosion and functional expansion - modelling and simulation method}

In order to associate robustness explosion and functional expansion with cost growth patterns, we introduce a simple functional cost model built on the following parameters and principles. Let us consider a system consisting of $n$ interconnected functionalities $F_{1}, \ldots, F_{n}$. The different functionalities $F_{1}, \ldots, F_{n}$ are improved as time $t$ increases. Let $U_{F_{i}}(t)$ be the cost required to develop the functionality $F_{i}$ individually, as an independent element from the system, at time $t$. The functionality $F_{i}$ is also connected to a percentage $p_{i}$ of the $n-1$ other functionalities. Let $I_{F_{i}}\left(t, p_{i}\right)$ be the cost related to the integration of $F_{i}$ within the system. For the simplicity of the simulation, in what follows, $p_{i}=p, \forall i=1 \ldots n$ where $p$ is a constant percentage which can be seen as the average level of connectedness of the system. Therefore, the total cost associated with the functionality $F_{i}$ at time $\mathrm{t}$ is $C_{F_{i}}(t)=U_{F_{i}}(t)+I_{F_{i}}(t, p)$. And the total development cost of the system is

$$
\sum_{1 \leq i \leq n} C_{F_{i}}(t)=\sum_{1 \leq i \leq n}\left[U_{F_{i}}(t)+I_{F_{i}}(t, p)\right]=U_{\text {total }}(t)+I_{\text {total }}(p, t)
$$

As time $t$ increases, some functionalities $F_{i}$ are required to be upgraded. And the engineering department faces a first challenge that is the obsolescence of the required knowledge to individually develop one functionality: we will describe this challenge with the function $\gamma_{1, i}(t)$ affecting the functionality $F_{i}$. However, for the simplicity of our simulation, $\gamma_{1, \mathrm{i}}(t)=\gamma_{1}(\mathrm{t}) \forall i=1 \ldots n$. Since our purpose will be to simulate the 'minimum' cost increase that can be expected from a product modification, we can consider that we chose $\gamma_{1}(t)$ such that $\gamma_{1}(t)=\min _{1 \leq i \leq n} \gamma_{1, \mathrm{i}}(\mathrm{t}) \quad \forall t$. Under these conditions, the evolution of the cost to develop $F_{i}$ as an independent element can be modelled with the recurrent equation:

$$
U_{F_{i}}(t)=\frac{\gamma_{1}(t)}{\delta_{1}(t)} . \quad U_{F_{i}}(t-1)
$$


where $\delta_{1}(\mathrm{t})$ is a function representing the capacity of an engineering department to counter obsolescence, i.e. the capacity of en engineering department to update and extend its current knowledge regarding individual functionalities. It can be seen as a learning rate.

Besides, improving some individual functionalities in order to make them more robust is likely to generate undesirable interactions (emergence phenomena) between systems which must be managed by engineering departments. If we describe the magnitude of emergence phenomena with the function $\gamma_{2}(t)$, the evolution of integration costs can be modelled with the following equation:

$$
I_{\text {total }}(t, p)=\frac{\gamma_{2}(t)}{\delta_{2}(t)} . \quad I_{\text {total }}(t-1, p)
$$

where $\delta_{2}(t)$ describes the capacity of the engineering department to master emergence phenomena.

For $k=\{1,2\}$, the capacity of an engineering department to cope with the challenge of obsolescence or emergence depends on whether the ratio $\frac{\gamma_{k}(t)}{\delta_{k}(t)}$ is more or less than 1 .

We now consider the introduction of set of $m$ new functionalities $\left\{F_{n+1}, \ldots, F_{n+m}\right\}$ within the system. An engineering departments will have to both deal with the 'individual' development of the new functionalities and with the integration of the new functionalities within the existing system. We could use the extent to which the newly-introduced functionalities are more expensive than the average cost of the initially existing individual functionalities (which we call $U_{\text {average }}$ ) to characterize the engineering department's capacity to manage the individual development of new functionalities. However, the cost increase due to $m$ new functionalities is likely to weigh less than the cost due to the new interactions can amount up to $\frac{m^{*}(m+n-1)}{2}$. Therefore, since we want to simulate the 'minimum' cost increase that can be expected when modifying the product, we will use the extent to which the number of new interactions is 'contained' to characterize the engineering department's capability to manage the introduction of new functionalities. To that end, we introduce the parameter $r_{3}(t)$, a percentage which is such that number of new interactions is $r_{3}(t) \cdot \frac{m^{*}(m+n-1)}{2}$. In our simulation, $r_{3}(t)$ can take three values: low, medium or high percentage.

In subpart 4.2, we model the cost evolution patterns characterizing different scenarii depending on (i) the magnitude of obsolescence phenomena $\gamma_{1}(t)$ and the learning capability of the engineering department $\left(\delta_{1}(\mathrm{t})\right)$, (ii) the magnitude of emergence phenomena $\gamma_{2}(t)$ the capability of the engineering department to control it it $\left(\delta_{2}(\mathrm{t})\right)$ (iii) the introduction of new functionalities and $r_{3}(t)$, the capability to integrate them.

\subsection{Test of our model - case study method}

In order to address RQ3, we study growth cases observed during the development of commercial aircraft and we attempt to identify the scenario of our model which they correspond to.

\section{RESULTS}

\subsection{A phenomenon of functional expansion affecting the development of commercial aircraft}

We counted the accumulation number of paragraph modifications, additions and deletions in CS-25 and FAR-25 with time: the results are given by the two increasing curves below. 


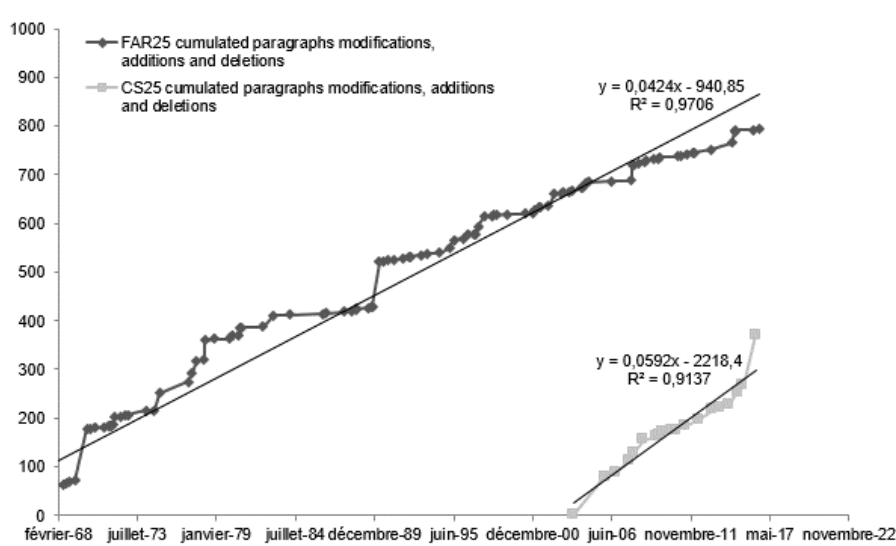

Figure 2. A steady growth in the number of regulatory changes affecting CS-25 and FAR-25

Focusing on the data collected on FAR-25 (because they feature a longer time span), we tested whether these trends correspond to a phenomenon of functional expansion. The endogenous regimes of functional expansion (mentioned in the literature review - 2.4) can be identified by representing the evolution of $r^{2}-r_{0}^{2}$ where $r$ is the rank of a matroid and by identifying whether or not the data fit with a linear regression. If they do, this means that we are in the face of an endogenous regime of functional expansion (Le Masson et al., 2019). An horizontal line corresponds to no expansion. The larger the slope of the regression line, the larger the magnitude of the endogenous functional expansion, and the greater the innovative design effort that is required on the part of the engineering department.

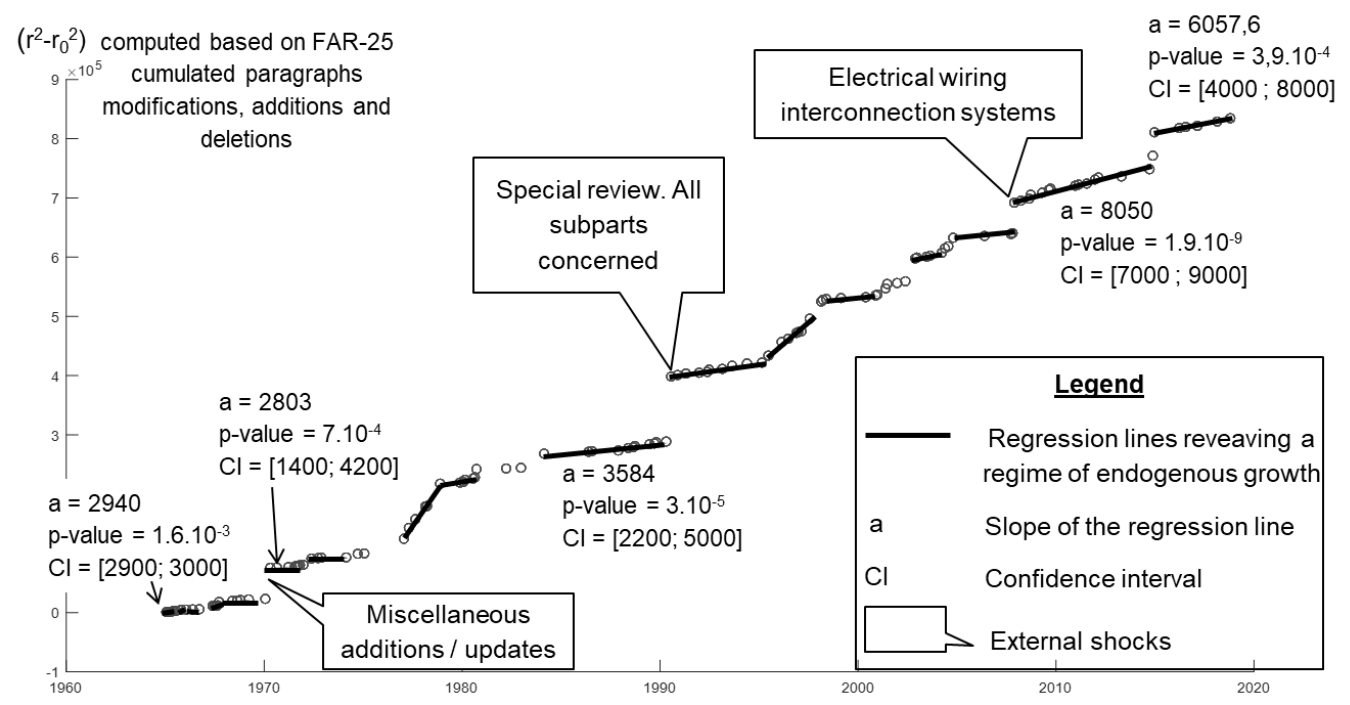

Figure 3. Identification of a slow endogenous regime regularly disturbed by external shocks

The results feature a regime of slow endogenous functional expansion regularly interrupted / disturbed by external shocks. The slope of the individual regression lines seems to increase with time, but verifying that the expansion regime indeed accelerates would require further statistical tests. Here, the results enable to answer RQ1: the development of commercial aircraft occur within an expanding functional space, implying an instable context involving both the frequent engineering improvements and frequent functional disruptions.

\subsection{Cost growth patterns associated with the management of robustness improvement and functional expansion}

\subsubsection{Absence of new functionalities}

In our simulation, we will model $\gamma_{1}(\mathrm{t}), \delta_{1}(\mathrm{t}), \gamma_{2}(\mathrm{t}), \delta_{2}(\mathrm{t})$ as linear functions. For $\mathrm{k}=\{1,2\}$, the relative positions of the lines representing $\gamma_{k}(t)$ and $\delta_{k}(t)$ represents the capacity of an engineering department to master the challenge (emergence or obsolescence). For $k=\{1,2\}$, the capacity of an 
engineering department to cope with the problem of obsolescence or emergence depends on whether the ratio $\frac{\gamma_{k}(t)}{\delta_{k}(t)}$ is more or less than 1 . The three different scenarii (S1., S2 and S3) can be distinguished:

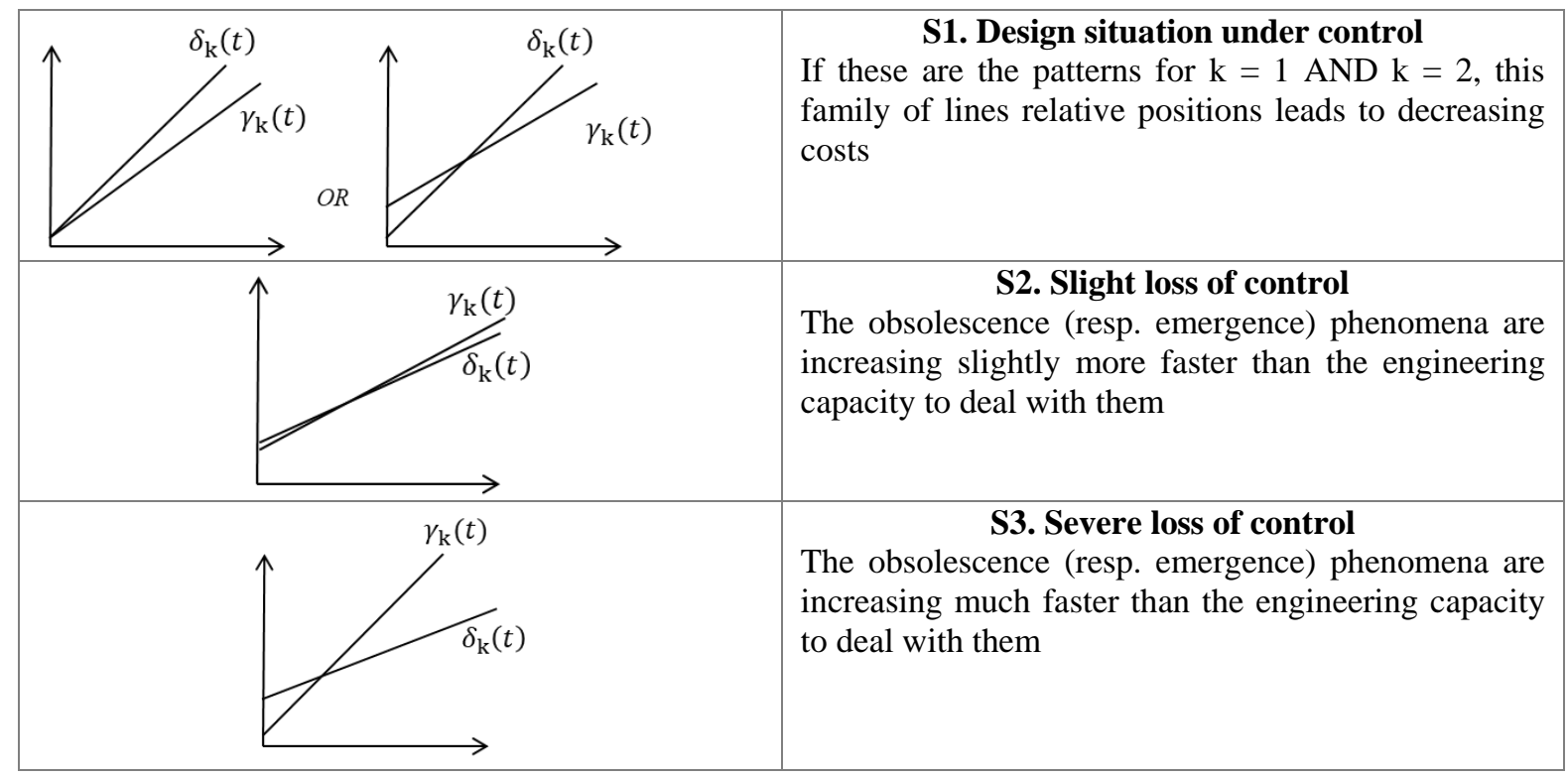

Note: in the figures that follow, the horizontal axis features time, the vertical axis features costs. As time increases, existing functionalities are regularly upgraded.

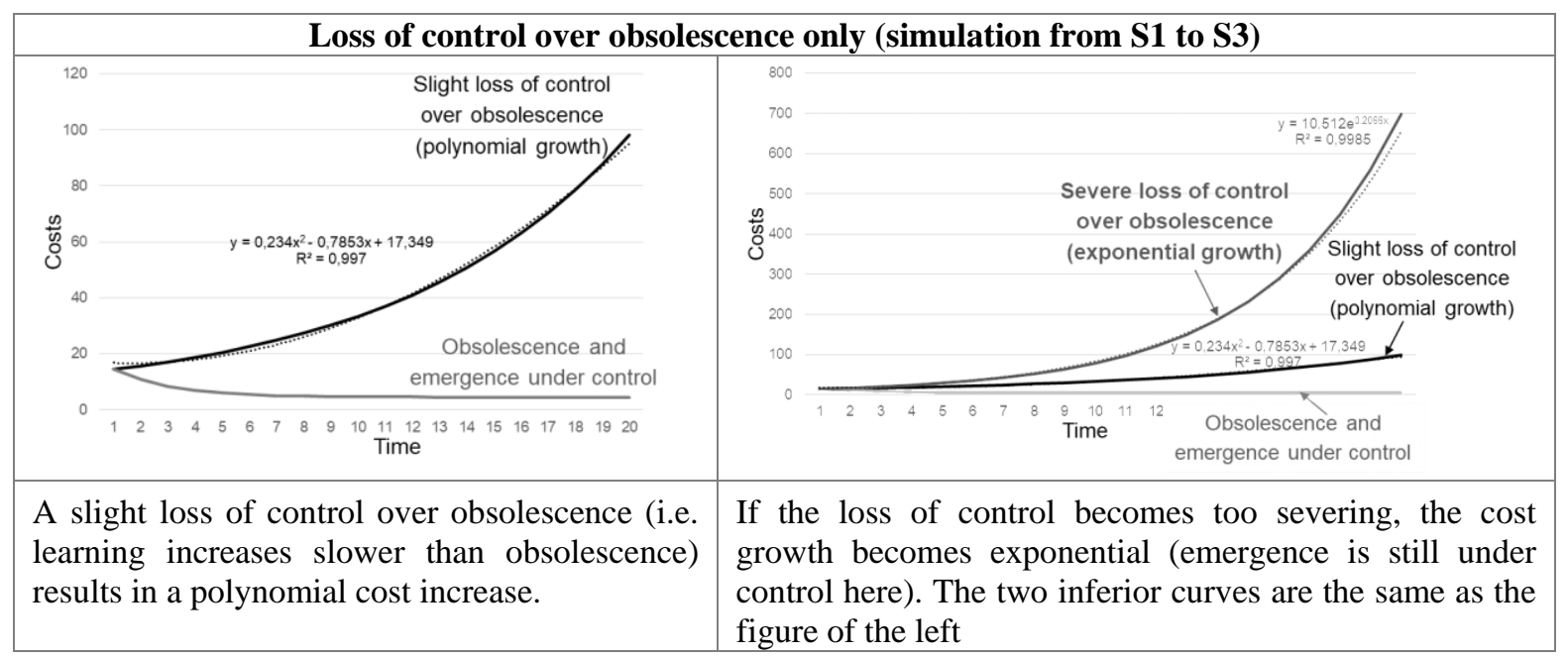

The simulation of a sole loss of control over emergence provides the same kind of results: as the loss of control becomes more dramatic, the trend shifts from polynomial to exponential. Unsurprisingly, given that emergence affects the interactions, which are more numerous than the functionalities, the threshold of the exponential growth is reached earlier with emergence phenomena. A loss of control alone can give rise to a superexponential growth. And unsurprisingly, combining both losses of control amplifies the cost growth phenomenon.

\subsubsection{Introduction of new functionalities}

We started by simulating a scenario in which the three aspects (obsolescence, emergence and introduction of new functionalities) were all out of control: we used a slight loss of control (S2.) for obsolescence and emergence. And we set . $r_{3}$ corresponding to the percentage of the new $\frac{m^{*}(m+n-1)}{2}$ possible new functionalities that have to be dealt with by designers is "high" $\left.(80 \%)\right)$. Such a scenario results in a super-exponential cost growth. 
Then, we attempted to identify the levers which could render the cost growth polynomial. The results are the following:

\begin{tabular}{|c|c|}
\hline 600000000 & 2500 \\
\hline 500000000 & \\
\hline ص 400000000 & \\
\hline की 300000000 & in 10 \\
\hline 200000000 & \\
\hline $\begin{array}{c}0 \longdiv { 1 3 5 7 9 1 1 1 3 1 5 1 7 1 9 2 1 2 3 2 5 2 7 2 9 } \\
\text { Time }\end{array}$ & 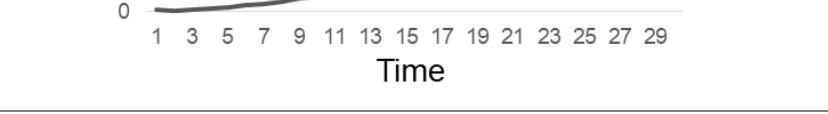 \\
\hline $\begin{array}{l}\text { Regaining control over obsolescence, even } \\
\text { unrealistically, with a } \frac{\gamma_{1}(\mathrm{t})}{\delta_{1}(\mathrm{t})} \text { ratio extremely } \\
\text { low, does not improve (at all) the } \\
\text { superexponential trend }\end{array}$ & $\begin{array}{l}\text { Significantly (unrealistically, with a } \frac{\gamma_{2}(\mathrm{t})}{\delta_{2}(\mathrm{t})} \text { ratio } \\
\text { extremely low) regaining control over emergence turns } \\
\text { the previous super-exponential growth into a polynomial } \\
\text { growth again }\end{array}$ \\
\hline
\end{tabular}

Or a last means to find back a polynomial cost growth is to set all control parameters at a reasonably good level of control.

\subsection{Test on empirically observed phenomena}

As a first test, we applied our model of the development cost trend followed by five successive programs launched by one aircraft manufacturer, over a period of 25 years. In order to eliminate from the comparison the possible impact resulting from economic factors (inflation, cost of material...), the costs were discounted so that they all reflect the same economic condition.

\begin{tabular}{|c|c|c|}
\hline 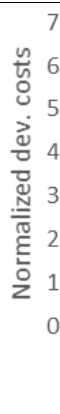 & $\begin{array}{c}y=0,2662 x^{2}-0,3247 x+1,1545 \\
R^{2}=0,9829\end{array}$ & $\begin{array}{l}\text { We observe a polynomial cost growth }\left(n^{2}\right) \\
\text { which reveals: } \\
\text { - either a reasonably good control of the three } \\
\text { design aspects that are: functionalities } \\
\text { individual upgrade, integration, and functional } \\
\text { expansion } \\
\text { - or an "uncontrolled" introduction of new } \\
\text { functionalities compensated by extremely } \\
\text { controlled integration capabilities }\end{array}$ \\
\hline
\end{tabular}

\section{CONCLUSION}

The considerable extent of the cost escalation trends presented in the introduction seems to highlight at first sight an alarming phenomenon, symptomatic of engineering departments having lost control over product development. By incorporating in a model three phenomena occurring at product level (1obsolescence phenomena ; 2-emergence phenomena ; 3- new functionalities) and three associated control parameters (controlled by designers), our model suggests that genuine 'out of control' situations should be characterized by exponential or even superexponential cost growth patterns. Therefore, our findings relativize the alarming character of the observed cost escalation. Put differently, given the dramatic extent demonstrated by products increase in robustness and functional expansion, the observed cost growth appear relatively well-contained. This suggests that engineering departments would own a 'hidden capability' which actually addresses very efficiently the challenges raised by transformations at product level, in particular phenomena of functional expansion. The different patterns of growth associated with the different scenarii could provide a firm with a diagnosis of its capacity not only to manage costs, but more importantly to manage robustness and functional expansion. However, these results are still at a nascent stage. We need to test the correctness of the diagnosis on additional cases and to refine the model in order to identify other possible cost patterns. 


\section{REFERENCES}

Alderson, D.L. and Doyle, J. (2010), “Contrasting Views of Complexity and Their Implications For NetworkCentric Infrastructures", IEEE Transactions on system man and cybernetics -Part A: systems and humans, Vol. 40 No. 4, July 2010.

Arena, M., Younossi, O., Brancato, K., Blickstein, I. and Grammich, C.A. (2008), "Why Has the Cost of FixedWing Aircraft Risen", A Microscopic Examination of the Trends in U.S. Military Aircraft Costs over the Past Several Decades. RAND (National Defense Research Institute and Project Air Force)

Augustine, N. R. (1983), “Augustine's Laws and major system development programs”, Revised and enlarged. 2nd ed. American Institute of Aeronautics / Astronautics, New York. ISBN: 0-915928-81-7.

Carlson, J.M. and Doyle, J.C. (1999), "Highly optimized tolerance: A mechanism for power laws in designed systems", Phys. Rev. E, Vol. 60 No. 1412.

Carlson, J.M., Doyle, J.C. (2002), “Complexity and robustness,” Proc. Nat. Acad. Sci. U.S.A., Vol. 99, pp. 2538-2545, Feb. 2002.

Dabkowski, M. and Valerdi, R. (2016), "Blockmodeling and the Estimation of Evolutionary Architectural Growth in Major Defense Acquisition Programs", Thirteenth Annual Acquisition Symposium.

de Weck, O.L. (2012), "Feasibility of a 5x speedup in system development due to META design", ASME/DETC2012-70791, 2012

El Qaoumi, K. (2015), “Mesurer l'innovation : modèles et études empiriques des changements d'identité des objets," (thèse), Mines ParisTech - PSL Research University, Sciences de Gestion, Paris.

Le Masson, P., El Qaoumi, K., Hatchuel, A. and Weil, B. (2018), "Functional expansion - eliciting the dynamics of consumer goods innovation with design theory". ICED.

Hove, K. and Lillekvelland, T. (2015), "Investment cost escalation - an overview of the literature and revised estimates".

Marshall, A.W. and William M. (1959), "Predictability of the Costs, Time, and Success of Development". Paper 1821. R and Corporation.

Sinha, K. (2014), "Structural complexity and its implications for design of cyber-physical systems", PhD Th, MIT.

Suh, E.S., Kim, I.Y. and de Weck, O. (2004), "Design for Flexibility: Performance and EconomicOptimization of Product Platform Components", 10th AIAA/ISSMO Multidisciplinary Analysis and Optimization Conference 30 August - 1 September 2004, Albany, New York

Suh, N.P. (2005), "Complexity: Theory and Applications", Oxford University Press, New York.

Winter, C. (2015), "A new approach to avoiding cost overruns and implementation delays in future large projects in aerospace business". Management Sciences Vol. 4 No. 25. 